\title{
AÇÕES DO PRÉ-NATAL REALIZADAS PELA EQUIPE DE ENFERMAGEM NA ATENÇÃO PRIMÁRIA À SAÚDE, CUIABÁ ${ }^{1}$
}

\section{ACTIONS PERFORMED BY THE NURSING TEAM IN PRENATAL CARE AT PRIMARY HEALTH CARE SETTING, CUIABÁ, BRAZIL}

\author{
ACCIONES REALIZADAS POR EL EQUIPO DE ENFERMERÍA EN LA \\ ATENCIÓN PRENATAL EN CENTROS DE ATENCIÓN PRIMARIA, \\ CUIABÁ, BRASIL
}

\author{
Sebastião Junior Henrique Duarte* \\ Marli Villela Mamede ${ }^{* *}$
}

\begin{abstract}
RESUMO
No Brasil a atenção qualificada ao pré-natal é uma das políticas de governo voltada às mulheres grávidas e desenvolvida pelos profissionais de saúde, entre eles o enfermeiro. Considerando as contribuições da enfermagem brasileira na redução da morbidade e mortalidade materna e infantil é que o estudo objetivou descrever as ações realizadas pela equipe de enfermagem na atenção pré-natal no município de Cuiabá, MT. Estudo descritivo participaram 182 profissionais da equipe de enfermagem, atuantes na Atenção Primária à Saúde de Cuiabá. Os dados foram coletados por meio da observação não participante e auxilio de um check-list contendo variáveis recomendadas pelo Ministério da Saúde e pela International Confederation of Midwives para atenção qualificada ao pré-natal. Utilizou-se a estatística descritiva para tabulação dos dados. O estudo foi aprovado pelo Comitê de Ética em Pesquisas da Universidade Federal de Mato Grosso sob número 694/2009. Os resultados revelaram baixa frequência das ações e competências essenciais ao pré-natal qualificado. Recomenda-se a elaboração e implementação de um protocolo de assistência pré-natal que possa nortear a consulta de enfermagem no pré-natal.
\end{abstract}

Palavras chave: Atenção Primária à Saúde, cuidado pré-natal, saúde da mulher, cuidados de enfermagem.

\begin{abstract}
In Brazil, specialized attendance at prenatal care is one of government policies addressed to pregnant women and developed by health professionals, including nurses. Considering the contributions of Brazilian nursing in reducing morbidity and maternal and infant mortality is that the study aimed to describe the actions taken by the nursing staff in prenatal city of Cuiabá, MT. A descriptive study involved 182 professionals of the nursing team, working in Primary Health Care in Cuiabá. Data were collected through non-participant observation and assistance of a check-list containing variables recommended by the Ministry of Health and the International Confederation of Midwives for specialized attendance at prenatal care. We used descriptive statistics for data tabulation. The study was approved by the Federal University of Mato Grosso Ethics Research Committee under number 694/2009. The results showed a low frequency of actions and practical essential competencies for qualified prenatal care. The development and implementation of a protocol for prenatal care to give direction to nursing consultation in prenatal care, is recommended.
\end{abstract}

Key words: Primary health Care, prenatal care, women's health, nursing care.

\footnotetext{
${ }^{1}$ Artigo abstraído da tese de doutorado intitulada: Estudo das competências essenciais na atenção pré-natal: ações da equipe de enfermagem no município de Cuiabá-MT, apresentada ao Programa Interunidades de doutoramento da Universidade de São Paulo, Escola de Enfermagem de Ribeirão Preto, 2010.

* Enfermeiro. Professor Universidade Federal de Mato Grosso do Sul, campus Três Lagoas. E-mail: sjhd@usp.br

${ }^{* *}$ Enfermeira. Professora Universidade de São Paulo, Escola de Enfermagem de Ribeirão Preto. E-mail: mavima@eerp.usp.br
} 


\section{RESUMEN}

En Brasil, la atención especializada del cuidado prenatal es una de las políticas gubernamentales dirigidas a las mujeres embarazadas y desarrollado por profesionales de la salud, incluyendo los profesionales de enfermería. Motivados por las contribuciones de la enfermería brasileña en la reducción de la morbilidad y la mortalidad materna e infantil es que el objetivo de este estudio se direccionó a describir las acciones realizadas por el personal de enfermería en el control prenatal de la ciudad de Cuiabá, MT. Se trata de un estudio descriptivo donde participaron de 182 profesionales del equipo de enfermería, que trabajan en Atención Primaria de Salud en Cuiabá. Los datos fueron recolectados a través de una observación no participante y la aplicación de una lista de chequeo que contenía las variables recomendadas por el Ministerio de Salud y la Confederación Internacional de Matronas para la atención especializada en el Control prenatal. Se utilizó estadística descriptiva para el análisis de los datos. El estudio fue aprobado por el Comité de Ética en Investigación de la Universidad Federal de Mato Grosso con el número 694/2009. Los resultados mostraron una baja frecuencia de las acciones y competencias prácticas esenciales en la atención prenatal calificada. Se recomienda el desarrollo e implementación de un protocolo para la atención prenatal para guiar la consulta de enfermería en el control prenatal.

Palabras clave: Atención Primaria de Salud, atención prenatal, salud de la mujer, cuidado de enfermería.

Fecha recepción: 20/11/11 Fecha aceptación: 19/03/13

\section{INTRODUÇÃO}

O pré-natal qualificado $(1,2)$ é aquele em que a mulher grávida e o recém-nascido recebem atendimento adequado durante a gravidez, o trabalho de parto, o parto, o período pós-parto e o neonatal, independente do local de atendimento: quer seja no domicílio, no centro de saúde ou no hospital. A gestante e sua família devem receber o cuidado não limitado apenas a procedimentos clínicos (3), mas um conjunto de ações com vistas à promoção de sua saúde, por meio da educação em saúde, do acolhimento, do vínculo de confiança, entre outras tecnologias de forma a desenvolver a autonomia da mulher para o seu auto cuidado.

A atenção adequada pode evitar importantes desfechos negativos na mãe e no recém-nascido, como complicações obstétricas, retardo no crescimento intrauterino, baixo peso ao nascer e prematuridade, contribuindo para a diminuição não só da morbidade como da mortalidade materna e infantil (4).

No entanto, a atenção à saúde da mulher na gestação permanece como um desafio tanto no que se refere à qualidade da assistência propriamente dita, quanto aos aspectos relacionados ao debate filosófico em torno do cuidado (5). O reconhecimento de que a atenção pré-natal, de um modo geral, não tem conseguido modificar resultados obstétricos e neonatais tem levado cada vez mais os pesquisadores a acreditarem que tal situação resulta de um conjunto de problemas interligados entre eles inclui a carência de profissionais qualificados (4).

A literatura aponta que a atenção qualificada ao pré-natal requer a implementação de procedimentos e ações específicos para esta fase de vida da mulher e que as ações dos profissionais responsáveis pelo pré-natal devam estar em consonância com as competências essenciais em obstetrícia, cujas diretrizes são descritas pelo Ministério da Saúde e pela International Confederation of Midwives (ICM) (5-7) e recomendadas pela Associação Brasileira de Enfermeiras Obstétricas e Obstetrizes-ABENFO (8).

A enfermagem brasileira tem dado importantes contribuições na redução da morbi-mortalidade materna e infantil e em várias 
localidades as enfermeiras têm se responsabilizado pela atenção pré-natal de gestantes de risco habitual $(9,10)$. No entanto os indicadores de mortalidade desse binômio no país ainda registram números elevados, os quais podem ser reduzidos por meio do cuidado qualificado.

Dados do município de Cuiabá, MT, registrados pelo Departamento de Informática do Sistema Único de Saúde (11) apontam que no ano de 2009 tiveram três óbitos maternos e outros dois ocorridos em 2010. Mesmo com a redução no número de mortes maternas estudo realizado em tal localidade (1), revelou que a equipe de enfermagem está comprometida com o cuidado pré-natal. Contudo um conjunto de fragilidades foi identificado revelando a necessidade de investimentos na organização e qualificação da assistência de enfermagem em Cuiabá, MT, a fim de oferecer atenção qualificada no prénatal.

Portanto, acredita-se que a atenção qualificada no pré-natal é essencial para redução da morbidade e mortalidade materna. Neste sentido, conhecer o que os profissionais de enfermagem fazem na atenção pré-natal possibilita identificar as ações realizadas, verificar a existência de correspondência de suas ações com as competências essenciais preconizadas pela ICM (2) e Organização Mundial da Saúde (12) (OMS), identificando fortalezas e fragilidades do serviço na promoção da saúde materna e neonatal no município estudado.

Então, conhecer a relação existente entre as ações dos profissionais de enfermagem e as competências essenciais para a atenção prénatal pode ser um indicador para a melhoria da qualidade dos serviços de pré-natal.

Tomando a relevância da temática e na intenção de contribuir com a sistematização da assistência de enfermagem qualificada ao pré-natal é que o estudo teve por objetivo descrever as ações realizadas pela equipe de enfermagem na atenção pré-natal no município de Cuiabá, MT.

\section{MÉTODO}

Estudo descritivo, exploratório, transversal, de abordagem quantitativa, desenvolvido nas unidades de saúde da Atenção Primária à Saúde (APS) da cidade de Cuiabá, capital do Estado de Mato Grosso, realizado de janeiro a dezembro de 2009. A população do município é de cerca de 580 mil habitantes. O número de nascidos vivos no ano de 2007 foi de 10.082, e a mortalidade geral hospitalar nesse mesmo ano foi de 1.966 óbitos, destes, 811 eram mulheres (13). A rede de saúde municipal contava com 63 equipes da Estratégia Saúde da Família (ESF), 22 Centros de Saúde (CS), 8 policlínicas com atendimento 24 h, 6 policlínicas odontológicas, 1 hospital geral municipal de grande porte e 1 laboratório, além das outras instituições públicas e privadas.

Foram considerados elegíveis para o estudo os profissionais de enfermagem que estivessem atuando na atenção pré-natal nos dois modelos de atenção (ESF e CS), há pelo menos um mês no período de coleta de dados.

Participaram do estudo 182 profissionais de enfermagem: 108 do nível médio, onde 60 são da ESF e 48 dos CS, e 74 enfermeiros, sendo 55 da ESF e 19 dos CS. Esses números correspondem ao quantitativo de profissionais que atuava no pré-natal, excluindo as perdas de 5 enfermeiros da ESF e 13 dos CS.

Os critérios de exclusão foram: não atuar diretamente na assistência pré-natal, recusa expressa em participar do estudo ou a critério do pesquisador depois de, no mínimo, três tentativas sem sucesso para o agendamento da coleta dos dados.

Os procedimentos para a coleta dos dados foram de duas formas: a) observação sistemática da atenção pré-natal oferecida pelos profissionais de enfermagem, tipo não participante, a qual foi subsidiada por um instrumento tipo check-list; b) entrevista semi estruturada com os profissionais de enfer- 
magem que assistiram as gestantes durante as observações, com vistas a colher dados de caracterização e a experiência profissional na atenção pré-natal. $O$ instrumento tipo check-list foi construído tomando por base o documento sobre as competências essenciais na atenção pré-natal da ICM (2) e manuais de atenção ao pré-natal do Ministério da Saúde do Brasil (6) cujos documentos identificam os fatores de interesse principais do estudo: as competências essenciais e normas para atenção pré-natal.

Os dados foram analisados utilizandose o Software Statistical Package for Social Sciences (SPSS). Os resultados foram organizados através de estatística descritiva por meio de índices absolutos e percentuais, e apresentados em tabelas.

O estudo foi aprovado pelo comitê de ética em pesquisas do Hospital universitário Julio Muller, expresso pelo protocolo 694/2009.

\section{RESULTADOS}

No período de coleta de dados foram observadas 74 atendimentos de gestantes, dos quais todas as pré-consultas foram realizadas pelos técnicos ou auxiliares de Enfermagem cujas ações se restringiram à: aferição da pressão arterial, verificação do peso corporal e medida da estatura. Aos enfermeiros coube a consulta de pré-natal propriamente dita.

Dentre as ações realizadas pelos enfermeiros as mais frequentes foram: preenchimento do cartão da gestante, tanto de primeira consulta como nas consultas subsequentes $(100 \%)$; realização do exame físico geral $(63,5 \%)$ e obstétrico $(48,6 \%)$ que constou da palpação abdominal, ausculta da frequência cardíaca fetal e mensuração da altura uterina. No entanto outras ações recomendadas pelo Ministério da Saúde brasileiro apresentaram baixa frequência como as orientações prestadas às gestantes, conforme ilustrado na Tabela 1.

Tabela 1. Orientações informadas às 74 gestantes pelos enfermeiros da Atenção Primária à Saúde do município de Cuiabá-MT. Cuiabá, 2009.

\begin{tabular}{|c|c|c|c|c|c|c|}
\hline \multirow{2}{*}{ Orientações fornecidas } & \multicolumn{2}{|c|}{$\begin{array}{c}\text { CS } \\
n=19\end{array}$} & \multicolumn{2}{|c|}{$\begin{array}{c}\text { USF } \\
n=55\end{array}$} & \multicolumn{2}{|c|}{$\begin{array}{c}\text { TOTAL } \\
\mathbf{n}=74\end{array}$} \\
\hline & $\mathbf{n}^{\mathbf{o}}$ & $\%$ & $\mathbf{n}^{\mathbf{o}}$ & $\%$ & $\mathbf{n}^{\mathbf{o}}$ & $\%$ \\
\hline Local e serviços de atendimento ao parto & 1 & 5,3 & 41 & 74,5 & 42 & 56,7 \\
\hline Alimentação & 7 & 36,8 & 25 & 45,5 & 32 & 43,2 \\
\hline Agendamentos: consultas e exames & 0 & 0 & 29 & 52,7 & 29 & 39,1 \\
\hline Quando procurar o serviço saúde & 2 & 10,5 & 22 & 40 & 24 & 32,4 \\
\hline Uso de medicamentos & 3 & 15,8 & 17 & 30,9 & 20 & 27,0 \\
\hline Medidas de conforto & 3 & 10,5 & 13 & 23,6 & 16 & 21,6 \\
\hline Sinais de perigo na gestação & 3 & 15,8 & 13 & 23,6 & 16 & 21,6 \\
\hline Cuidados com o recém-nascido & 0 & 0 & 13 & 23,6 & 13 & 17,5 \\
\hline Cuidados com as mamas & 3 & 15,8 & 8 & 14,5 & 11 & 14,8 \\
\hline Aleitamento materno & 2 & 10,5 & 5 & 9,1 & 7 & 9,4 \\
\hline Sinais de trabalho de parto & 1 & 5,3 & 6 & 10,9 & 7 & 9,4 \\
\hline Atividade física durante a gestação & 1 & 5,3 & 5 & 9,1 & 6 & 8,1 \\
\hline Planejamento familiar & 2 & 10,5 & 4 & 7,3 & 6 & 8,1 \\
\hline Sono e repouso na gestação & 2 & 10,5 & 4 & 7,3 & 6 & 8,1 \\
\hline
\end{tabular}


Continuação Tabela 1.

\begin{tabular}{llccccc}
\hline Modificações corporais & 0 & 0 & 5 & 9,1 & 5 & 6,7 \\
Fumo, álcool e drogas ilícitas & 2 & 10,5 & 2 & 3,6 & 4 & 5,4 \\
Higiene materna & 2 & 10,5 & 1 & 1,8 & 3 & 4,0 \\
Sexualidade & 1 & 5,3 & 2 & 3,6 & 3 & 4,0 \\
Atividade laboral & 0 & 0 & 2 & 3,6 & 2 & 2,7 \\
Uso de preservativos & 0 & 0 & 1 & 1,8 & 1 & 1,3 \\
\hline
\end{tabular}

No que se refere às orientações quanto ao uso de preservativos durante as relações sexuais, aos cuidados com o recém-nascido, atividade laboral e agendamento de consultas e exames, apenas os Enfermeiros da ESF ofereceram tais informações às gestantes.

Durante a coleta dos dados foi possível verificar que, para as gestantes que estavam na primeira consulta pré-natal, os enfermeiros solicitaram os seguintes exames laboratoriais e de diagnóstico: grupo sanguíneo, fator $\mathrm{Rh}$, hemograma, glicemia, urina tipo I, parasitológico de fezes, teste anti-HIV, sorologias (Hepatite A, Hepatite B, Hepatite C, Rubéola, Sífilis, Toxoplasmose, Citomegalovírus e Clamídia), Coombs indireto, lipidograma e BAAR e cultura de escarro (Tabela 2).

Tabela 2. Exames solicitados para 74 gestantes atendidas pelos enfermeiros da Atenção Primária à Saúde do município de Cuiabá-MT. Cuiabá, 2009.

\begin{tabular}{|c|c|c|c|c|c|c|}
\hline \multirow{2}{*}{ Exames solicitados } & \multicolumn{2}{|c|}{$\begin{array}{c}\text { CS } \\
n=19\end{array}$} & \multicolumn{2}{|c|}{$\begin{array}{c}\text { USF } \\
n=55\end{array}$} & \multicolumn{2}{|c|}{$\begin{array}{c}\text { TOTAL } \\
\mathrm{n}=74\end{array}$} \\
\hline & $\mathbf{n}^{\mathbf{o}}$ & $\%$ & $\mathbf{n}^{\mathbf{o}}$ & $\%$ & $n^{\circ}$ & $\%$ \\
\hline Urina tipo I & 6 & 31,6 & 23 & 41,8 & 29 & 39,1 \\
\hline Glicemia & 5 & 26,3 & 23 & 41,8 & 28 & 37,8 \\
\hline Hemograma & 5 & 26,3 & 23 & 34,5 & 28 & 37,8 \\
\hline Sorologia para Sífilis & 5 & 26,3 & 23 & 41,8 & 28 & 37,8 \\
\hline Teste anti-HIV & 5 & 26,3 & 22 & 40 & 27 & 36,4 \\
\hline Sorologia para Toxoplasmose & 5 & 26,3 & 21 & 38,2 & 26 & 35,1 \\
\hline Sorologia para Hepatite B & 5 & 26,3 & 19 & 34,5 & 24 & 32,4 \\
\hline Sorologia para Rubéola & 5 & 26,3 & 19 & 34,5 & 24 & 32,4 \\
\hline Fator Rh & 6 & 31,6 & 17 & 25,5 & 23 & 31,0 \\
\hline Grupo sanguíneo & 6 & 31,6 & 17 & 25,5 & 23 & 31,0 \\
\hline Ultrassonografia & 0 & 0 & 8 & 14,5 & 8 & 10,8 \\
\hline Parasitológico de fezes & 2 & 10,5 & 7 & 12,7 & 9 & 12,1 \\
\hline Citomegalovírus & 1 & 5,3 & 3 & 5,5 & 4 & 5,4 \\
\hline Lipidograma* $^{*}$ & 0 & 0 & 2 & 3,6 & 2 & 2,7 \\
\hline Sorologia para Hepatite C & 1 & 5,3 & 1 & 1,8 & 2 & 2,7 \\
\hline BAAR e cultura de escarro & 0 & 0 & 1 & 1,8 & 1 & 1,3 \\
\hline Clamidea & 0 & 0 & 1 & 1,8 & 1 & 1,3 \\
\hline Coombs indireto & 0 & 0 & 1 & 1,8 & 1 & 1,3 \\
\hline Sorologia para Hepatite A & 0 & 0 & 1 & 1,8 & 1 & 1,3 \\
\hline
\end{tabular}

${ }^{\star}$ Colesterol total, Colesterol frações, Triglicerídeos. 
Do total de mulheres grávidas observadas, 27 eram consultas de primeira vez, dessas três gestantes já portavam resultados de exames no momento da consulta. Excluindo essas três gestantes, restou um total de 24 consultas de primeira vez para serem solicitados os exames.

Observa-se que a maioria dos exames laboratoriais foi solicitada para $100 \%$ das gestantes de primeira consulta, pelos enfermeiros dos serviços estudados. Com relação à ultrassonografia, foi requisitada para 10,8\% das gestantes de primeira consulta. Observou-se que quando o enfermeiro indica a realização da ultrassonografia, preenche o formulário de pedido do exame e leva para o médico assinar, pois no município de Cuiabá os enfermeiros não são habilitados a solicitar tal exame, mesmo assim, alguns enfermeiros não deixaram as gestantes desassistidas da ultrassonografia. Constatou-se também que, nos dois modelos estudados, a coleta de material para exame colpocitológico não foi efetuada em nenhuma consulta, durante o período de coleta de dados.

Uma enfermeira solicitou exames (BAAR e cultura de escarro) para monitorização do tratamento da Tuberculose em uma gestante. Essa gestante já estava em acompanhamento pré-natal de alto risco e frequentava, também, a unidade básica de saúde da família.

Vale destacar que nem todos os profissionais que solicitaram exames, no período de observação deste estudo, explicaram o motivo da realização desses exames a todas as gestantes.

Ainda em relação aos exames, algumas gestantes compareceram nas consultas de pré-natal já com resultados, principalmente nas consultas subsequentes, como mostra a Tabela 3.

Tabela 3. Exames interpretados durante o atendimento de 74 gestantes atendidas pelos enfermeiros da Atenção Primária à Saúde do município de Cuiabá-MT. Cuiabá, 2009.

\begin{tabular}{lrrrrrc}
\hline & \multicolumn{2}{c}{ CS } & \multicolumn{2}{c}{ USF } & \multicolumn{2}{c}{ TOTAL } \\
Exames interpretados & \multicolumn{2}{c}{$\mathbf{n = 1 9}$} & \multicolumn{2}{c}{$\mathbf{n = 5 5}$} & \multicolumn{2}{c}{$\mathbf{n = 7 4}$} \\
\cline { 2 - 7 } & $\mathbf{n}^{\mathbf{0}}$ & $\mathbf{9}$ & $\mathbf{n}^{\mathbf{0}}$ & $\mathbf{9}$ & $\mathbf{n}^{\mathbf{0}}$ & \% \\
\hline Urina tipo I & 5 & 26,3 & 14 & 25,5 & 19 & 25,6 \\
Hemograma & 5 & 26,3 & 12 & 21,8 & 17 & 22,9 \\
Teste anti-HIV & 3 & 15,8 & 9 & 16,4 & 12 & 16,2 \\
Fator Rh & 6 & 31,6 & 5 & 9,1 & 11 & 14,8 \\
Grupo sanguineo & 6 & 31,6 & 5 & 9,1 & 11 & 14,8 \\
Glicemia & 3 & 15,8 & 7 & 12,7 & 10 & 13,5 \\
Sorologia para Sífilis & 2 & 10,5 & 8 & 14,5 & 10 & 13,5 \\
Sorologia para Toxoplasmose & 3 & 15,8 & 7 & 12,7 & 10 & 13,5 \\
Sorologia para Hepatite B & 2 & 10,5 & 7 & 12,7 & 9 & 12,1 \\
Sorologia para Rubéola & 2 & 10,5 & 7 & 12,7 & 9 & 12,1 \\
Parasitológico de fezes & 1 & 5,3 & 5 & 9,1 & 6 & 8,1 \\
Citomegalovírus & 0 & 0 & 3 & 5,5 & 3 & 4,0 \\
Lipidograma* & 0 & 0 & 3 & 5,5 & 3 & 4,0 \\
Ultrasonografia obstétrica & 0 & 0 & 3 & 5,5 & 3 & 4,0 \\
Sorologia para Hepatite A & 0 & 0 & 1 & 1,8 & 1 & 1,3 \\
Sorologia para Hepatite C & 0 & 0 & 1 & 1,8 & 1 & 1,3 \\
Cálcio sérico & 0 & 0 & 1 & 1,8 & 1 & 1,3 \\
\hline
\end{tabular}

${ }^{\star}$ Colesterol total, Colesterol frações, Triglicerídeos. 
Apesar de alguns exames apresentados pelas gestantes não fazerem parte da rotina do pré-natal, os enfermeiros interpretaram e ofereceram as orientações pertinentes.

De acordo com o Programa de Humanização no Pré-natal e Nascimento do Ministério da Saúde, os exames da rotina pré-natal devem ser solicitados na primeira consulta e repetidos ao longo da gestação, especialmen- te o hemograma, VDRL e urina tipo I.

Os achados sobre os medicamentos prescritos, durante a consulta pré-natal, pelos enfermeiros, mostram que os medicamentos Sulfato Ferroso e Ácido Fólico, foram prescritos com maior frequência, enquanto os medicamentos como: Buscopan, Paracetamol e Plasil tiveram menor frequência de prescrição, conforme aponta a Tabela 4.

Tabela 4. Medicamentos prescritos pelos enfermeiros da Atenção Primária à Saúde do município de Cuiabá-MT, durante 74 consultas de pré-natal observadas. Cuiabá, 2009.

\begin{tabular}{|c|c|c|c|c|c|c|}
\hline \multirow[t]{2}{*}{ Medicamentos prescritos } & \multicolumn{2}{|c|}{$\begin{array}{c}\text { CS } \\
n=19\end{array}$} & \multicolumn{2}{|c|}{$\begin{array}{c}\text { USF } \\
\mathrm{n}=55\end{array}$} & \multicolumn{2}{|c|}{$\begin{array}{c}\text { TOTAL } \\
\mathbf{n}=74\end{array}$} \\
\hline & $\mathbf{n}^{\mathbf{o}}$ & $\%$ & $\mathbf{n}^{\mathbf{o}}$ & $\%$ & $n^{\circ}$ & $\%$ \\
\hline Ácido Fólico e Sulfato Ferroso & 0 & 0 & 17 & 30,9 & 17 & 22,9 \\
\hline Sulfato Ferroso & 2 & 10,3 & 4 & 7,3 & 6 & 8,1 \\
\hline Paracetamol & 0 & 0 & 2 & 3,6 & 2 & 2,7 \\
\hline Buscopam & 0 & 0 & 1 & 1,8 & 1 & 1,3 \\
\hline Plasil & 0 & 0 & 1 & 1,8 & 1 & 1,3 \\
\hline
\end{tabular}

Analisando tais achados de acordo com o modelo de atendimento, verificou-se que os enfermeiros dos CS não prescrevem medicamentos rotineiramente. A prescrição de medicamentos por enfermeiros em Cuiabá-MT ainda não está claramente definida e, diante de queixa clínica, a maioria dos enfermeiros encaminha a gestante para avaliação e conduta médica.
De acordo com resultados apresentados na Tabela 5, verifica-se que indicação com maior frequência para a prescrição dos medicamentos é para inclusão da gestante no Programa Saúde de Ferro do Ministério da Saúde, que recomenda o uso do Sulfato Ferroso para profilaxia e tratamento da Anemia e o Ấcido Fólico que atua na formação do tubo neural fetal.

Tabela 5. Motivos da prescrição de medicamentos pelos enfermeiros da Atenção Primária à Saúde do município de Cuiabá-MT, durante 74 consultas de pré-natal observadas. Cuiabá, 2009.

\begin{tabular}{|c|c|c|c|c|c|c|}
\hline \multirow[t]{2}{*}{ Motivos da prescrição dos medicamentos } & \multicolumn{2}{|c|}{$\begin{array}{c}\text { CS } \\
n=19\end{array}$} & \multicolumn{2}{|c|}{$\begin{array}{c}\text { USF } \\
\mathrm{n}=55\end{array}$} & \multicolumn{2}{|c|}{$\begin{array}{c}\text { TOTAL } \\
\mathbf{n}=74\end{array}$} \\
\hline & $\mathbf{n}^{\mathbf{o}}$ & $\%$ & $\mathbf{n}^{\mathbf{o}}$ & $\%$ & $\mathbf{n}^{\mathbf{o}}$ & $\%$ \\
\hline Inclusão no Programa Saúde de Ferro & 2 & 10,3 & 21 & 38,2 & 23 & 31 \\
\hline Cefaléia & 0 & 0 & 2 & 3,6 & 2 & 2,7 \\
\hline Cólica & 0 & 0 & 2 & 3,6 & 2 & 2,7 \\
\hline Náuseas e/ou vômitos & 0 & 0 & 2 & 3,6 & 2 & 2,7 \\
\hline
\end{tabular}


Os dados revelam, também, que 2,7\% das gestantes tiveram prescritos pelos enfermeiros medicamentos para cefaléia, cólica e náuseas e vômitos, no entanto somente dois enfermeiros da ESF procederam à prescrição.

Constatou-se, ainda, durante a observação das consultas, que $27 \%$ dos profissionais explicaram o motivo da prescrição medicamentosa e orientaram as gestantes quanto ao uso da medicação.

Os resultados da Tabela 6 apresentam as atividades de desfecho realizadas pelos enfermeiros durante a consulta, como segue: encaminhamento para vacina antitetânica $(29,7 \%)$, encaminhamento para Papanicolaou $(9,4 \%)$, agendamento das consultas subsequentes $(40,5 \%)$ e anotações no prontuário e cartão da gestante (72,9\%).

Tabela 6. Atividades de desfecho realizadas ao final de 74 consultas de pré-natal feitas pelos enfermeiros da Atenção Primária à Saúde do município de Cuiabá-MT. Cuiabá, 2009.

\begin{tabular}{|c|c|c|c|c|c|c|}
\hline \multirow{2}{*}{ Atividades } & \multicolumn{2}{|c|}{$\begin{array}{c}\text { CS } \\
n=19\end{array}$} & \multicolumn{2}{|c|}{$\begin{array}{c}\text { USF } \\
n=55\end{array}$} & \multicolumn{2}{|c|}{$\begin{array}{c}\text { TOTAL } \\
\mathrm{n}=74\end{array}$} \\
\hline & $\mathrm{n}^{\mathbf{o}}$ & $\%$ & $\mathrm{n}^{\mathbf{o}}$ & $\%$ & $\mathbf{n}^{\mathbf{o}}$ & $\%$ \\
\hline Anotação no prontuário e cartão da gestante & 14 & 73,7 & 53 & 96,4 & 54 & 72,9 \\
\hline Agendamento de consulta subsequente & 0 & 0 & 30 & 54,5 & 30 & 40,5 \\
\hline Encaminhamento para sala de vacina & 3 & 15,8 & 19 & 34,5 & 22 & 29,7 \\
\hline Orientação retorno para puerpério & 3 & 15,8 & 7 & 12,7 & 10 & 13,5 \\
\hline Encaminhamento para Papanicolaou & 0 & 0 & 7 & 12,7 & 7 & 9,4 \\
\hline Anotação somente no prontuário & 3 & 15,8 & 2 & 3,6 & 5 & 5,7 \\
\hline Orientação sobre vacinação e motivos & 0 & 0 & 4 & 7,3 & 4 & 5,4 \\
\hline Encaminhamento para dentista & 1 & 5,3 & 2 & 3,6 & 3 & 4 \\
\hline Orientação em atividades em grupo de gestantes & 1 & 5,3 & 2 & 3,6 & 3 & 4 \\
\hline Encaminhamento para nutricionista & 1 & 5,3 & 1 & 1,8 & 2 & 2,7 \\
\hline
\end{tabular}

Do total de 74 gestantes consultadas, 35 já tinham sido vacinadas ou estavam em dia com a vacina antitetânica.

Dentre as atividades realizadas, ilustradas na Tabela 6, observa-se que a anotação/registro no prontuário e cartão da gestante foi efetuada em $72,9 \%$. Ressalta-se que todas as gestantes portavam o cartão da gestante no momento da consulta.

$\mathrm{O}$ agendamento das consultas subsequentes foi orientado em 40,5\% do total das gestantes. As consultas subsequentes são agendadas na recepção das unidades estudadas e constatou-se que muitas das gestantes já conheciam a rotina desses agendamentos. Com relação ao encaminhamento das ges- tantes para o exame de Papanicolaou pelos enfermeiros, os dados mostram que apenas $9,4 \%$ das gestantes foram orientadas a fazer o exame. Durante a coleta dos dados ficou evidente que a realização do exame Papanicolaou não é uma prática de consenso entre os enfermeiros de Cuiabá-MT, há aqueles que recomendam e outros que contra indicam tal exame no período gestacional, mesmo para gestantes com queixa ginecológica.

Constatou-se que os enfermeiros encaminharam $5(6,7 \%)$ gestantes para avaliação e conduta médica, frente a queixas ginecológicas (dor no baixo ventre, suspeita de perda sanguínea e Leucorréia). Observou-se, também, que das 74 gestantes atendidas, 3 
(4\%) foram encaminhadas para o dentista e $2(2,7 \%)$ foram encaminhadas para avaliação com nutricionista, respectivamente.

Quanto às atividades de educação em saúde, observou-se durante a coleta dos dados que houve ações educativas em apenas três unidades da ESF, duas ocorreram na sala de espera e uma na sala de reuniões. Em relação ao acolhimento, na maioria das vezes foi realizado durante as consultas.

\section{DISCUSSÃO E CONCLUSÕES}

A análise da assistência pré-natal realizada pelos profissionais de enfermagem, na rede municipal de saúde do município de Cuiabá-MT, revelou diferenças nas ações e procedimentos desenvolvidos pelos enfermeiros e pelos profissionais de enfermagem de nível médio cabendo aos últimos as ações de menor complexidade. Revelou também diferenças nas ações dos enfermeiros nos dois modelos de atenção (CS e ESF), identificando a necessidade de novos estudos para compreender tais diferenças.

Os achados revelaram que não há padronização quanto aos exames que devem ser requisitados às gestantes, cada profissional orienta-se por referenciais que vão desde o Manual do Pré-natal do Ministério da Saúde (6) às experiências adquiridas durante a formação profissional. Desse modo, identificou-se que alguns exames são solicitados sem indicação, como o exame de sorologia para a Hepatite A em gestante assintomática, e outros que fazem parte da rotina do prénatal deixam de ser solicitados para algumas gestantes, como a sorologia para Hepatite C.

Diante dessas observações, cabe reforçar que as normas e os protocolos são fundamentais para guiar e respaldar uma atenção qualificada por parte dos profissionais de enfermagem, assim como para medir o desempenho e promover um sentido de responsabilidade profissional(2).
O exame de Parasitológico das fezes foi requisitado somente para $12,1 \%$ das gestantes, limitando o diagnóstico de verminose em mais de $85 \%$ das gestantes. É fundamental tratar as parasitoses de todas as pessoas, especialmente das gestantes, evitando complicações como o déficit na absorção dos nutrientes. Deve ser considerado que o Programa Saúde de Ferro (13) recomenda o uso do Sulfato Ferroso e do Ácido Fólico na gestação, quando verminoses interferem na absorção de tais medicamentos.

As sorologias para Hepatite B e C são importantes na gestação para medidas que evitam a transmissão vertical, semelhante ao HIV. O Coombs indireto deve ser avaliado sempre que a gestante tiver fator Rh negativo. Outros exames que foram solicitados, como a sorologia para Citomegalovírus, o Lipidograma, a Clamídia, o BAAR e a cultura de escarro, mostram que em algumas situações o atendimento de enfermagem não está restrito aos requisitos mínimos recomendados, sendo investigadas outras morbidades.

Dos exames solicitados nas consultas subsequentes, a Sorologia para Hepatite B foi o mais frequente. De acordo com o Programa de Humanização no Pré-natal e Nascimento (14), os exames de rotina pré-natal devem ser repetidos próximo a $30^{\mathrm{a}}$ semana de gestação, especialmente as sorologias para HIV, Sífilis e urina tipo I, não apenas a Hepatite B.

Do total de gestantes que compareceram na primeira consulta de pré-natal, cerca de $30 \%$ já portavam resultados de exames, principalmente as que foram atendidas nos CS, sendo assim, do ponto de vista biológico, parte da avaliação das condições de saúde das gestantes foi adequadamente conduzida.

Em ambos os modelos de atenção (CS e ESF) houve gestantes com resultados de exames nas consultas subsequentes. Uma das facilidades para a realização dos exames está no fato de que o município de Cuiabá-MT dispõe de laboratório municipal e a realização dos exames é rápida, tendo em vista que o agendamento e coleta de material para os 
exames são feitos na maioria das unidades de saúde.

Ficou evidente que no modelo CS a prescrição de medicamentos não é facultada aos Enfermeiros, enquanto no ESF é uma prática frequente nos atendimentos de pré-natal por enfermeiros, principalmente o Sulfato Ferroso e o Ácido Fólico, ambos normatizados pelo Programa Saúde de Ferro do Ministério da Saúde (13), no qual 45,5\% das gestantes foram cadastradas na primeira consulta.

Os medicamentos em uso foram verificados em 48,9\% das gestantes, no sentido de certificar se elas faziam uso de Sulfato Ferroso e Ácido Fólico. Tais medicamentos foram os prescritos com maior frequência pelos enfermeiros, levando a considerar que as recomendações do Programa Saúde de Ferro (13) foram incorporadas na prática dos enfermeiros. Em relação ao uso de medicamentos pelas mulheres grávidas, a ICM (2) recomenda a possibilidade de inclusão de plantas medicinais e outras preparações não farmacológicas pelas gestantes, principalmente para o alívio do desconforto. Durante o período de coleta de dados não se observou nenhuma atitude por parte dos enfermeiros no sentido de investigar não somente o uso destes recursos, como também a restrição do uso de medicamento na gestação.

Ao final dos atendimentos das consultas de pré-natal observou-se que em $29,7 \%$ dos atendimentos ocorreu o encaminhamento para a sala de vacinação e, para $5,4 \%$ das gestantes, foi explicado o motivo de tal procedimento. Quanto ao agendamento das consultas subsequentes, 40,5\% de todas as gestantes foram orientadas sobre a data do retorno para a próxima consulta, que foi registrado no cartão da gestante e no livro de agendamentos das consultas de pré-natal, revelando que essas mulheres grávidas tiveram assegurada a consulta de retorno; para aquelas que já estavam próximo da data do parto, o agendamento seguinte ocorreu semanalmente.

A avaliação da situação vacinal ocorreu na maioria das consultas de pré-natal, em- bora devesse ocorrer para todas as gestantes. Das gestantes com história vacinal incompleta, os enfermeiros as encaminhavam para a sala de vacinação, na qual os profissionais de nível médio se responsabilizavam pelo procedimento.

Com relação ao encaminhamento para o parto, os enfermeiros referiram que não há necessidade de encaminhamento específico, basta que cada gestante procure um dos hospitais que atendem ao parto. Observouse que a maioria dos enfermeiros informou para as gestantes o nome e endereço dos hospitais, porém, tal conduta não garante que as gestantes sejam atendidas no parto, revelando uma fragmentação da assistência à mulher grávida ao final da gestação, o que pode acarretar em sérias consequências para a mãe e para o bebê.

Os registros das informações foram apontados no prontuário e no cartão da gestante sendo que, em uma unidade de saúde, além do prontuário individual, havia uma cópia do cartão da gestante, mantendo os mesmos registros, o que revela uma preocupação dos enfermeiros com o acompanhamento da evolução da gestação.

Apesar das dificuldades e fragilidades dos profissionais de enfermagem no desempenho das competências essenciais na atenção pré-natal, e seguimento das normas do Ministério da Saúde a implantação do atendimento pré-natal pelos enfermeiros foi efetivada na rede básica de Cuiabá. Contudo, os profissionais da equipe de enfermagem que participaram do estudo referiram gosto pela atuação no pré-natal e, de modos distintos, atendem a clientela que necessita do acompanhamento gestacional, dando importante contribuição às gestantes cuiabanas.

Dificuldades quanto a condução da atenção pré-natal por enfermeiros de Rio Branco foram também identificadas por outros autores $(5,7)$, especialmente quanto à compreensão de resultados de exames laboratoriais e utilização de normas e protocolos.

Estudo realizado na rede pública munici- 
pal de Rio Grande, que analisou o uso das normas do pré-natal estabelecidas pelo Ministério da Saúde, encontrou um desempenho superior da ESF comparado ao modelo tradicional, em alguns procedimentos, mas também identificou que existem outros que estão aquém do valor esperado, mostrando que as práticas dos profissionais de saúde na atenção ao pré-natal devem continuar sendo aperfeiçoadas (4).

Da mesma forma, o presente estudo mostrou que não há definição de condutas estabelecidas em protocolos assistenciais, ficando evidente que as ações desenvolvidas na assistência ao pré-natal pela equipe de enfermagem não são padronizadas e orientadas por práticas baseadas em evidências científicas, comprometendo, assim, a atenção qualificada ao pré-natal.

Os padrões e protocolos definem o tipo de cuidado oferecido em cada nível do sistema de saúde e a contribuição de cada categoria de provedor é essencial para orientar e apoiar a prática da atenção qualificada no ciclo gravídico puerperal, assim como para avaliar e melhorar o desempenho dos provedores. Da mesma forma, as normas e diretrizes também podem ser utilizadas como base para auto avaliação na prática clínica por parte do pessoal qualificado e para avaliações de programas de supervisão por pares (2). Vale ressaltar que o pré-natal integra as rotinas do trabalho dos enfermeiros, sendo assegurado pela Lei do exercício profissional (15).

Contudo, quando se pretende melhorar a capacidade dos profissionais para proteger e promover a saúde materna, infantil e comunitária é indispensável estabelecer programas de capacitação de alta qualidade e de supervisão. A participação de enfermeiros e enfermeiras obstétricas tem fundamental importância para o fortalecimento da assistência pré-natal, entretanto, outros estudos também revelam que, são necessários investimentos na formação de pessoal qualificado, para o atendimento à mulher no ciclo grávi- do-puerperal, a fim de melhorar a qualidade de atendimento nos serviços de saúde materna e neonatal (16-18).

Chama-se a atenção para que um ambiente favorável ao desenvolvimento de pessoas capacitadas implica, entre outras coisas, a existência de associações profissionais que promovam e defendam uma atenção de alta qualidade durante a gestação (2). Nesta direção, a Associação Brasileira de Obstetrizes e Enfermeiras Obstétricas (ABENFO) tem exercido um papel importante na elaboração de políticas nacionais de formação e atenção à mulher, e de normas técnicas baseadas em evidência científicas e de perfis de competências básicas no atendimento à mulher e ao neonato para enfermeiros e obstetrizes (8).

Esse estudo apontou a necessidade da (re)definição, padronização e avaliação das ações desenvolvidas pela equipe de enfermagem em Cuiabá-MT, o que, evidentemente, propiciará o fortalecimento da assistência prestada às mulheres grávidas e, com isso, a melhoria na assistência pré-natal.

Embora o município não conte com protocolo assistencial que oriente as consultas de enfermagem no pré-natal, ficou evidente que os enfermeiros inseridos no modelo de atenção Estratégia Saúde da Família-ESF atuam com mais presença e autonomia na assistência às gestantes comparados aos enfermeiros dos Centros de Saúde. Portanto, a participação dos enfermeiros no pré-natal, em Cuiabá-MT, ocorre distintamente ao comparar os dois modelos de atenção à saúde, o que pode limitar a participação da enfermagem no controle e redução da mortalidade materna.

Recomenda-se a colaboração das Associações de Classe como ABENFO e ABEN, e das universidades na qualificação dos profissionais de enfermagem para o atendimento pré-natal e estabelecimento de protocolos e perfis profissionais que atendam às exigências para o desempenho das competências essenciais no atendimento à mulher e à família em processo de gestação. Recomendase, ainda, que as instituições de ensino dis- 
cutam e aprimorem o modelo de formação profissional pautado no ensino por competências, em que os futuros profissionais possam apreender o conhecimento, praticar as habilidades e adquirir atitudes requeridas no cuidado com as gestantes.

\section{REFERÊNCIAS}

1. Duarte SJH. Estudo das competências essenciais na atenção pré-natal: ações da equipe de enfermagem no município de Cuiabá-MT. [Tese de doutorado em Ciências da Saúde com ênfase em enfermagem]. Universidade de São Paulo. Ribeirão Preto, 2010.

2. International Confederation of Midwives. Essential Competencies for Basic Midwifery Practice 2010. ICM, 2011. Disponível em: http://www.internationalmidwives.org

3. Duarte SJH, Andrade SMO. Assistência pré-natal no Programa Saúde da Família. Esc Anna Nery. 2006; 10: 121-125.

4. Mendonza-Sassi RA, Cesar JÁ, Teixeira TP, Ravache C, Araújo GD, Silva TC. Diferenças entre unidades da Estratégia Saúde da Família e unidades tradicionais em um município da Região Sul do Brasil. Cad Saude Pública. 2011; 27(4): 78796.

5. Costa GD, Cotta RMM, Reis JR, Batista RS, Gomes AP, Franceschini SCC. Avaliação do cuidado à saúde da gestante no contexto do Programa Saúde da Família. Cienc Saude Coletiva. 2009; 14(suplem): 1347-357.

6. Ministério da Saúde (BR). Pré-natal e puerpério. Atenção qualificada e humanizada. Brasília, 2006. CUNHA, Margarida de Aquino 2012.

7. Cunha MA, Mamede MV, Dotto LMG, Araruna RC. Assistência pré-natal por profissionais de enfermagem no municí- pio de Rio Branco, Acre, Amazônia. Rev. Bahiana de Saúde Pública. 2012; 36(1): 174-90.

8. Mamede MV, Dotto LMG, Oliveira ME, Bruggemann O, Riesgo MLG, Gualda DMR, Arantes SL. Minuta do Concurso para Certificação e Titulação em Enfermagem Obstétrica e Neonatal pela Associação Brasileira de Enfermagem Obstétrica e Neonatal. Rio de Janeiro, 2008. Disponível em: http://abenfo.redesindical.com.br

9. Cunha MA, Mamede MV, Mamede FV. Assistência pré-natal: competências essenciais desempenhadas por enfermeiros. Esc. Anna Nery. 2009; 13(1): 145-53.

10. Duarte SJH, Mamede MV. Estudo das competências essenciais na atenção prénatal: ações da equipe de enfermagem no município de Cuiabá-MT. Enferm Foco. 2012; 3(3): 75-80.

11. Brasil. Ministério da Saúde. Departamento de Informática do Sistema Único de Saúde (DATASUS). 2013. [citado 06 abr 2013]. Disponível em http://www2. datasus.gov.br

12. Organização Mundial da saúde. Maternidade segura. Assistência ao parto normal: um guia prático. Genebra: OMS, 1996.

13. Cuiabá. Secretaria Municipal de Saúde. Diretoria da Atenção Básica. Cuiabá, 2010.

14. Brasil. Ministério da Saúde. Manual operacional: Programa nacional de suplementação de Ferro. Brasília, DF, 2005.

15. Brasil. Ministério da Saúde. Programa humanização do parto: humanização no pré-natal e nascimento. Brasília, DF, 2002.

16. Brasil. Presidência da República Federativa do Brasil. Lei no. 7.498, de 25 de jun. de 1986. Dispõe sobre a regulamentação do exercício da Enfermagem e dá outras providências. Diário Oficial da União. Brasília, 26 jun 1986, seção I p. 9.2739275. 
17. Lourenção DCA, Bentido GAV. Competências gerenciais na formação do enfermeiro. Rev Bras Enferm. 2010; 63 (1): 9197.
18. Shimizu HE, Lima MG. As dimensões do cuidado pré-natal na consulta de enfermagem. Rev Bras Enferm. 2009; 62(3): 387-392. 